\title{
Lattice Dynamics of Laser Excited Ferroelectric $\mathrm{BaTiO}_{3}$
}

\author{
D. Issenmann, S. Schleef, S. Ibrahimkutty, G. Buth, T. Baumbach, A. Plech \\ Institute for Synchrotron Radiation, KIT, Postfach 3640, D-76021 Karlsruhe, Germany \\ M. BEyER AND J. DEMSAR \\ Center for Applied Photonics, University of Konstanz, Universitätsstr. 10, D-78457 Konstanz, Germany
}

\begin{abstract}
We investigated the lattice dynamics of the prototypic ferroelectric barium titanate close to its ferroelectric-paraelectric phase transition aiming at a better understanding of the atomistic nature of the transition. The usage of time-resolved X-ray techniques allows to disentangle lattice motion and unit cell changes, which, in part, relate to the ferroelectric polarization. In the quasi-static case both the electrical and the laser excitation show a mean-field, simple thermal behaviour, while for time scales shorter than nanoseconds the impulsive nature of the excitation becomes visible.
\end{abstract}

PACS: 78.47.D-, 61.05.cp, 77.22.Ej, 65.40.De, 64.60.Cn

\section{Introduction}

Ferroelectrics have been under investigation since 1920 and still attain an increasing relevance in applications $[1,2]$. One of the most commonly analyzed examples is barium titanate $\left(\mathrm{BaTiO}_{3}\right)$; it is the first discovered prototype for the class of perovskite ferroelectrics [3]. Therefore, it is interesting to notice that even in this long-discussed representative not all seemingly basic processes have been fully uncovered yet. To be specific, the phase transition from the ferroelectric tetragonal phase to the paraelectric cubic phase is a perpetual subject of discussion. While the macroscopic behavior of the compound at the phase transition is clear, it is not yet fully understood which processes contribute to what extent to this phase transition on a microscopic scale.

The main question is whether the phase transition to the ferroelectric phase with the spontaneous electrical polarization as order parameter is caused predominantly by a displacement of the titanium ions relative to the center of the unit cell (which is called a displacive phase transition [4]) or if the underlying process is the statistical distribution of the permanent displacement of the titanium ions from the cell center. A long range order of this displacement then leads to a microscopic net polarization (thus commonly named order-disorder phase transition [5]).

The order-disorder mechanism was discussed since the findings of diffuse sheets of scattering intensity, which were attributed to chains of ordered Ti displacement [5-7]. The success of the soft-mode theory, however, put the system as a textbook example for a displacive type transition, until recent research with microscopic tools (e.g. absorption spectroscopy or X-ray diffuse scat- tering [8]), have underlined the earlier results (e.g. the Raman scattering) that disorder is still present in the high-symmetry cubic phase. This led to the conclusion that the phase transition has an important order-disorder nature. The structure involved with the disorder is an uncorrelated displacement in the $\langle 111\rangle$ direction, which sums up to a net $\langle 100\rangle$ displacement in the low-symmetry (tetragonal) phase [9].

Some very recent publications [10] doubt this approach, pointing out that a very prominent soft mode branch of the transversal optical modes associated with the titanium movement within the oxygen cage can be fully responsible for the apparent disorder in the high-symmetry (cubic) phase. What makes the discussion even more difficult is that this soft mode is actually overdamped [11], thus approaching static disorder.

To shed light on this phenomenon we have (i) studied non-thermal excitation pathways, such as laser excitation, and (ii) used a microscopic probe, as X-ray scattering, in order to resolve the atomic scale dynamics [12-14].

\section{Methods}

Firstly, a series of experiments was performed to study the behavior of the barium titanate crystal structure when approaching the phase transition temperature $T_{\mathrm{c}}$ $\left(130^{\circ} \mathrm{C}\right)$ under the influence of a time-dependent electric field. For this purpose, a barium titanate sample with (100) orientation was placed in a heating holder; the temperature monitored via temperature sensors (Pt 100) was controlled by means of a temperature controller (model 332, Lakeshore). An alternating electrical field (modulated by a sine wave output of a function generator with a frequency of $250 \mathrm{kHz}$ ) was applied parallel to the direction of the poled crystal axis ( $c$-axis). The applied field 
and the polarization of the sample were monitored by an oscilloscope via a Sawyer-Tower circuit. This setup was placed on a six-circle diffractometer (beamline SCD at the ANKA synchrotron ring) to perform X-ray diffraction measurements using the (400) Bragg reflections. The diffracted radiation was detected by an avalanche photodiode (APD, Cyberstar-FMB Oxford) and recorded in a time-resolved manner synchronized to the period of the external electric field by means of a histogram card (Nanoharp, Picoquant) [15]. With this setup it was possible to measure the $E$-field correlated intensity as well as position of the Bragg peak for different temperatures. These are directly related to the structure factor and the lattice plane distance, respectively. In turn, the structure factor is an indication of the movement of the titanium relative to the symmetry position in the unit cell.

A second series of experiments was performed at the ID09B beamline at the ESRF ring in Grenoble [16, 17]. Time-resolved X-ray scattering was used to gather information down to a picosecond time scale. The experiments were carried out in a pump-probe scheme whereby a femtosecond titanium-sapphire $\left(\mathrm{Ti}: \mathrm{Al}_{2} \mathrm{O}_{3}\right)$ amplifier provided the pump pulse with a maximum energy of $2 \mathrm{~mJ}$ at a wavelength of $795 \mathrm{~nm}$. The pulses with a temporal width of 100 femtoseconds were delivered at a repetition rate of $1 \mathrm{kHz}$ to match the train of X-ray pulses from the synchrotron. The laser beam was focused to a diameter of $200 \mu \mathrm{m}$ at the sample plane. For the probe beam, the optics of the ID09B beamline provided an X-ray beam with an energy of $15 \mathrm{keV}$ ( $\mathrm{Si}$ (111) monochromatized) on a spot size of $100 \mu \mathrm{m}$. The scattered radiation was collected again by an APD detector (Cyberstar) and time-resolved by the histogram card with a time resolution of $4 \mathrm{~ns}$. Sample angle and detector angle could be varied in a $\theta-2 \theta$ configuration, while some $10^{4}$ shots were integrated per angular setting. The barium titanate crystal was mounted on a heater and the temperature was controlled by means of the temperature controller.

Optical spectroscopy has been performed at a $250 \mathrm{kHz}$ femtosecond amplifier system (RegA, Coherent) with degenerate pump and probe pulses $\left(\lambda_{0}=800 \mathrm{~nm}\right)$ at near normal incidence in transmission. An edge filter has been used near the laser center wavelength in order to enhance the contrast on the lattice induced transient optical changes as a Raman type probe signal is characterized by a slight shift in wavelength.

\section{Results of the quasistatic excitation with electrical field}

The electrical field excitation is applied in the limit of low perturbation, as the electrical field is considerably weaker than the coercitive field for domain reversal [18]. We applied $\pm 22 \mathrm{~V}$ across a $1 \mathrm{~mm}$ thick crystal, while a voltage of $500-1000 \mathrm{~V}$ is needed for domain switching. Thus only the susceptibility of the crystal is probed. The crystal is poled along the $z$ axis (perpendicular to the surface).
To analyze the results of these experiments the angularly resolved scattered intensities for each time step were fitted with a Lorentzian peak function. Firstly, a distortion of the barium titanate crystal following the alternating electrical field could be observed, showing a periodic angular shift of the Bragg peak (Fig. 1).

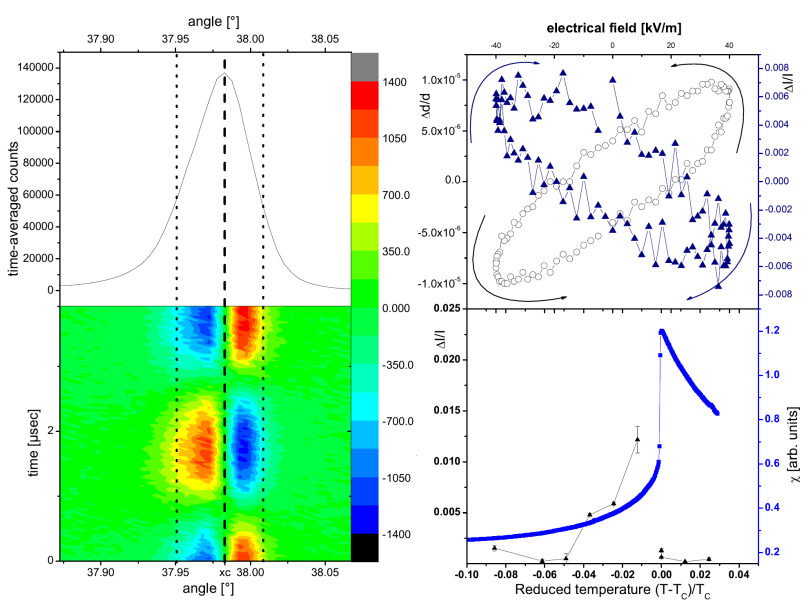

Fig. 1. Left: time average (upper half) and color-coded time-resolved difference map (lower half) of the $\mathrm{BaTiO}_{3}$ (400) Bragg peak when applying an electrical field (vertical axis). The dashed lines are drawn for orientation, "xc" designates the assumed central $x$-axis position, i.e. the angular center of the Bragg peak. Upper right: hysteresis loops of the change of the structure factor (triangles) and strain (circles) of the (400) peaks as function of the applied electrical field $4 \mathrm{~K}$ below the phase transition temperature. Lower right: magnitude of the structure factor change amplitude as function of the reduced temperature (triangles) together with the dielectric response $\chi$ of the crystal (squares).

To quantify this shift the peak angles for each Lorentzian fit curve were converted into lattice parameter changes using Bragg's law. By correlating the temporal behavior to the sinusoidal electrical field a hysteresis loop can be observed, which reveals the piezoelectricity. The data in Fig. 1 show that the strain is almost linear in the electrical field with only a small phase shift.

The area under the curve can be analyzed in a similar way. As the electrical field is weak in comparison to the coercitive field, the change of the integrated intensity can be entirely attributed to the change of the structure factor. Consequently, the X-ray scattering results can disentangle the lattice strain from the polarization change.

This structure factor is sensitive to the relative change of the atoms within the unit cell and thus to the electrical polarization. The loss of the phase information during the scattering process is the reason for only being able to determine the relative change of the atoms from their initial position, but not the absolute polarization direction. Such distinction would only be possible by anomalous scattering [19]. Assuming a model, where the (positively charged) titanium atom moves antiparal- 
lel to the oxygen atoms in the surrounding octaeder, one would expect a decrease of the integrated intensity with increasing deviation of the titanium from the cell center and thus increasing field along the polarization. This is indeed observed in the hysteresis loop for the intensity (see Fig. 1). The same calculation for the (430) peak of a (110) cut crystal would produce an increase of intensity, which is indeed found in the experiment (not shown).

It is instructive to follow the temperature behavior of these quantities. The amplitude of the change in lattice parameter increases with temperature when approaching the phase transition in the tetragonal phase, but falls steeply to zero when the phase transition is crossed. In other words, as soon as the ferroelectricity vanishes, i.e. the spontaneous polarization goes to zero, the piezoelectric effect also vanishes. This shows that in the high temperature phase the electrical field does not couple to the ferroelectric order, which is in agreement with both a displacive and an order-disorder transition. The dielectric measurements, however, typically show a large amplitude of the dielectric susceptibility even above the phase transition, as seen in the bottom right part of Fig. 1. This is well explained by the power law behavior in the basic Landau-Devonshire description, which predicts an inverse proportionality of the susceptibility both below and above the phase transition.

Assuming that the ionic mobility contributes the electrical susceptibility one expects that the structure factor would couple to the electrical field and its amplitude would increase towards the phase transition. This is indeed the case below $T_{\mathrm{c}}$, while above $T_{\mathrm{c}}$ no structure factor changes are observed anymore. This is at first surprising, but can be explained within the order-disorder picture and taking into account that a site change of the $\mathrm{Ti}$ atom may not change the absolute distance of the $\mathrm{Ti}$ atom from the cell center, but only its sign. Thereby it contributes to the electrical susceptibility, but not to the structure factor change as the latter is not sensitive to the sign.

\section{Laser-induced lattice changes}

The laser excitation has been performed with 60 fs pulses at $800 \mathrm{~nm}$, a wavelength which lies well below the band gap of $\mathrm{BaTiO}_{3}$. Therefore the pure thermal impact of the laser is small and restricted to some two-photon absorption. The interaction with below-bandgap excitation has been intensively studied, revealing an impulsive stimulated Raman scattering process (ISRS) for the excitation of the lattice. Dougherty and coworkers [20, 21] have shown that the selective excitation of transverse optical phonons shows the signature of mode softening close to the phase transition.

Indeed, in Fig. 2 we see that the transient changes in the optical pump-probe experiments do not show a typical femtosecond scale rise of the response due to electron excitation, but a smooth bimodal change of the absorption within the first picosecond. This is typical for the

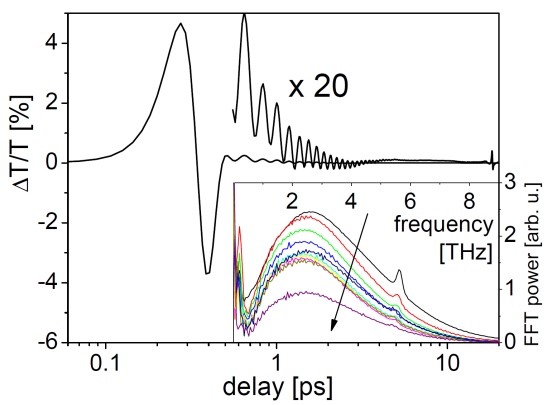

Fig. 2. Time-resolved optical transmission change through a crystal along the $\langle 100\rangle$ direction. The inset shows a Fourier spectrum of the transient transmission change.

ISRS type of scattering. Subsequent oscillations of some 200 fs period follow, which would decay within 15 ps. The power spectrum of the transient absorption shows a broad peak around $2-3 \mathrm{THz}$, which is identical to the overdamped excitation that had been observed earlier by $\mathrm{THz}$ spectroscopy [11]. Being both active in the Raman and infrared spectroscopy this excitation indicates the broken inversion symmetry below the phase transition. It undergoes a softening when approaching the phase transition. The long coherent oscillation results in a sharp peak in the Fourier spectrum (inset) at around 5.5 THz, which is caused by a mixed $\mathrm{LO}+\mathrm{TO}$ phonon. It is not considered central for the transition, but also shows some damping due to the changes in elasticity close to the transition temperature. Some signatures of a further very slow phonon motion are detected around $0.2 \mathrm{THz}$, which are, however, very difficult to interpret.

Therefore, we can expect to observe lattice changes, when performing a laser-pump and X-ray-probe experiment. This has been done in a similar way to the electrical measurements with a quasi-continuous X-ray source (a $15 \mathrm{keV}$ X-ray beam at the ID09 beamline of ESRF in hybrid filling mode) and by means of a histogramming card in order to cover a long time span after the excitation. Figure 3 shows the results of the Bragg scattering on a (110) oriented $\mathrm{BaTiO}_{3}$ crystal, when analyzing the peak from one of the domains, which are oriented with the long (polarization) axis at 45 degrees to the surface. The lattice parameter shift as well as the integrated intensity can be derived analogous to the electrical case above.

The typical reaction of the lattice is a fast expansion within the first $100 \mathrm{~ns}$, which then decays slowly towards the lattice parameter before excitation. This rise time is given by the finite expansion of the $1 \mathrm{~mm}$ thick crystal, which happens with the speed of sound. The relaxation on the $100 \mu$ s time scale is, however, artificial, as it is imposed by the $1 \mathrm{kHz}$ repetition rate. In case of a partial relaxation on this time scale the transient signal shows a relaxation time commensurate with the repetition rate of the experiment. 


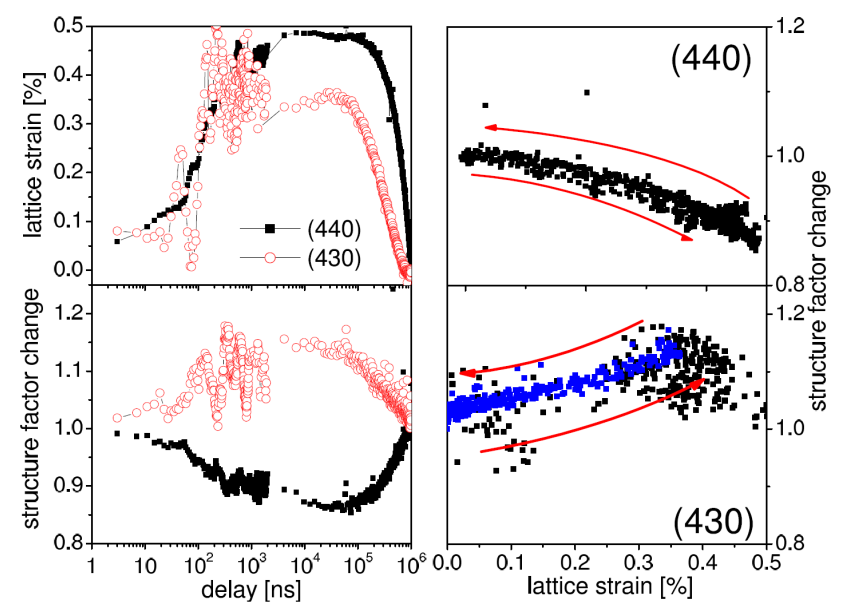

Fig. 3. Left: time-resolved changes of lattice parameter and structure factor of the Bragg reflections (440, top) and (430, bottom) as function of the delay after laser excitation $19^{\circ} \mathrm{C}$ below the phase transition. Right: structure factor - strain hysteresis loops of the (440) and (430) reflections.

More importantly, one can also observe the structure factor changes induced by the laser pulses. These contain new information, as the polarization is directly accessible. Since the Bragg angle change can be linked to strain and the intensity change can be linked to a structure factor change for the respective Bragg reflection, one can use a representation that shows the relation between the two following the laser irradiation. This view shows to be instructive in terms of characterization of the system behavior on short time scales since one may expect a difference between excitation and relaxation of strain and structure factor, which should be clearly visible as hysteretic curve in this view. The corresponding plots are shown in Fig. 3.

In fact, the curves show a decrease of the structure factor with strain for the (440) and an increase for the (430) reflection. This behavior is expected, when the excursion of the titanium (and oxygens) increases from the cell center. This displacement is, as seen above for the ISRS mechanism, as well as in an earlier study [13] not explained by a thermal excitation, but by an increase of the order parameter.

Apart from this non-thermal stimulation the hysteresis loop is rather closed, which indicates that the polarization closely follows the strain. Consequently on this time scale, which is long relative to the phonon time scales, polarization and strain are closely coupled and no further insight into the microscopic nature of the ferroelectricity is possible.

\section{Local tools}

The Bragg scattering shows the lattice expansion and the atomic movement within the unit cell via the change of the structure factor of the respective reflection. It should be kept in mind that the X-ray structure revealed by this approach is only an average one, which does not reflect the local disorder or the dynamics of the phonons, unless a very high time resolution is used. Furthermore, the loss of phase information has a serious impact on the derivation of the titanium displacement. As the change of the structure factor shows only the mean square displacement of titanium and oxygen atoms from the unit cell center, the Bragg scattering cannot distinguish between locally ordered or disordered atoms. This means that one cannot discern between a fully ordered polarization in the ferroelectric phase and a local disorder according to the 8-site model [9]. In this aspect it is clear that the results from the Bragg scattering, at least on the nanosecond time scale and longer, show a behavior, which is in agreement with the simple mean-field approach.

There are, however, local X-ray tools available, which can potentially give complementary information. As Ravel et al. [8] have shown, X-ray absorption spectroscopy can be used as a tool to determine the local structure surrounding the titanium atom by probing its $K$ edge. In particular the near-edge structure (XANES) is sensitive on the symmetry breaking of the $\mathrm{Ti}-\mathrm{O}$ bonds and therefore reports on a $\mathrm{Ti}$ displacement. Advantage can also be taken from the fact that the X-ray emission at synchrotrons is linearly polarized. Therefore one can distinguish the direction of the symmetry breaking, when measuring the $s-d$ transition, which is dipole forbidden, but will be allowed for the broken symmetry [8]. Still the XANES exhibits a similar phase problem, as only the symmetry breaking, but not its direction can be obtained.

The order-disorder contribution can particularly be addressed by X-ray diffuse scattering. Indeed, already very early it had been reported that $\mathrm{BaTiO}_{3}$ and related compounds show particularly weak distributions of diffuse scattering close to the Bragg reflections. This diffuse scattering even increases for the high-symmetry phases, which is a strong indication for the disorder even in the cubic phase. A reaction of the diffuse scattering and the XANES on the laser excitation would therefore deliver new information. One should nevertheless keep in mind that a high time resolution will be important as well to record these changes on a time scale which matches the phonon lifetimes in the system. A time resolution below 100 ps should therefore be envisaged.

\section{Conclusions}

The structural response of a ferroelectric system, $\mathrm{BaTiO}_{3}$, to external stimuli has been explored in order to better understand the mechanism of the ferroelectricity. X-ray scattering as an atomic scale probe can disentangle the pure lattice change, namely the piezoelectric response, from the atomic scale modifications, which are seen by a variable structure factor. Both the electrical field and laser excitation show these unit cell changes, which are in agreement with an enhancement of the ferroelectric order parameter (given by the Ti displacement) 
with electrical field and laser excitation, respectively. It is confirmed that the laser excitation mechanism is a Raman type excitation, which is strongly enhanced by the stimulated emission of phonons by the bandwidth of the femtosecond laser pulses.

The results on the Bragg scattering on a nanosecond time scale can be regarded as a quasistatic excitation, where the polarization closely follows the lattice strain. In order to learn more about the microscopic nature of the transition we plan to use local tools such as time-resolved XANES and diffuse scattering.

\section{Acknowledgments}

The work is supported by the DFG by individual grant and by the Heisenberg fellowship. We would like to acknowledge beam time at ESRF with the help from M. Wulff and L. Guérin and at ANKA.

\section{References}

[1] J.F. Scott, C.A.P. de Araujo, Science 246, 1400 (1989).

[2] J.F. Scott, Science 315, 954 (2007).

[3] H.F. Kay, P. Vousden, Philos. Mag. 40, 1019 (1949).

[4] W. Cochran, Adv. Phys. 9, 387 (1960).

[5] R. Comes, M. Lambert, A. Guinier, Solid State Commun. 6, 715 (1968).

[6] R. Comes, M. Lambert, A. Guinier, Acta Crystallogr. A 26, 244 (1970).

[7] S. Ravy, J.-P. Itié, A. Polian, M. Hanfland, Phys. Rev. Lett. 99, 117601 (2007).

[8] B. Ravel, E.A. Stern, R.I. Vedrinski, V. Kraizman, Ferroelectrics 206-207, 407 (1998).

[9] R. Pirc, R. Blinc, Phys. Rev. B 70, 134107 (2004).
[10] E.G. Maksimov, N.L. Matsko, J. Exp. Theor. Phys. 108, 435 (2009).

[11] J. Hlinka, T. Ostapchuk, D. Nuzhnyy, J. Petzelt, P. Kuzel, C. Kadlec, P. Vanek, I. Ponomareva, L. Bellaiche, Phys. Rev. Lett. 101, 167402 (2008).

[12] C. v. Korff Schmising, M. Bargheer, M. Kiel, N. Zhavoronkov, M. Woerner, T. Elsaesser, I. Vrejoiu, D. Hesse, M. Alexe, Phys. Rev. Lett. 98, 257601 (2007).

[13] K. Istomin, V. Kotaidis, A. Plech, Q. Kong, Appl. Phys. Lett. 90, 022905 (2007).

[14] H. Navirian, H. Enquist, R. Nüske, A. Jurgilaitis, C. v. Korff Schmising, P. Sondhauss, J. Larsson, Phys. Rev. B 81, 024113 (2010).

[15] S. Ibrahimkutty, D. Issenmann, S. Schleef, A.-S. Müller, Y.-L. Mathis, B. Gasharova, E. Huttel, R. Steininger, J. Göttlicher, T. Baumbach, A. Bartels, C. Janke, A. Plech, J. Synchr. Rad. 18, 539 (2011).

[16] F. Schotte, S. Techert, P.A. Anfinrud, V. Srajer, K. Moffat, M. Wulff, in: Third-Generation Hard X-ray Synchrotron Radiation Sources, Ed. D. Mills, Wiley, Berlin 2002

[17] M. Cammarata, L. Eybert, F. Ewald, W. Reichenbach, M. Wulff, P.A. Anfinrud, F. Schotte, A. Plech, Q. Kong, M. Lorenc, B. Lindenau, J. Räbiger, S. Polachowski, Rev. Sci. Instrum. 80, 15101 (2009).

[18] E. Zolotoyabko, J.P. Quintana, B.H. Hoerman, B.W. Wessels, Appl. Phys. Lett. 80, 3159 (2002).

[19] S.J. van Reeuwijk, K. Karakaya, H. Graafsma, S. Harkema, J. Appl. Cryst. 37, 193 (2004).

[20] T.P. Dougherty, G.P. Wiederrecht, K.A. Nelson, M.H. Garrett, H.P. Jensen, C. Warde, Science $\mathbf{2 5 8}$ 770 (1992).

[21] T.P. Dougherty, G.P. Wiederrecht, K.A. Nelson, M.H. Garrett, H.P. Jenssen, C. Warde, Phys. Rev. B 50, 8996 (1994). 